\title{
Visualization of Large Ontologies with Landmarks
}

\author{
Zong Lei Jiao ${ }^{1}$, Qiang Liu ${ }^{1}$, Yuan-Fang $\operatorname{Li}^{1}$, Kim Marriott ${ }^{1,2}$ and Michael Wybrow ${ }^{1,2}$ \\ ${ }^{1}$ Faculty of Information Technology, \\ Monash University, Clayton, Victoria 3800, Australia \\ ${ }^{2}$ National ICT Australia, Victoria Laboratory \\ $\{$ zljial,qliu40\}@student.monash.edu \\ \{YuanFang.Li,Kim.Marriott,Michael.Wybrow\}@monash.edu
}

Keywords: Ontology: Visualization: Interaction

\begin{abstract}
Ontologies are building blocks of the Semantic Web and are essential for knowledge representation and data integration. Interactive visualization is an important tool for the understanding of ontologies, especially large ones. In this paper, we present a novel hierarchical ontology visualization method for effectively visualizing an ontology's global landmarks, local structure and individual class' details. We have implemented a visualization system that can handle large ontologies efficiently. Preliminary evaluation indicates that the method and the system can be effectively used to understand large ontologies.
\end{abstract}

\section{Introduction}

Ontologies underpin the Semantic Web. They describe domain knowledge using classes (abstract concepts), predicates (binary relationships) and individuals (concrete instances). A class represents a set of individuals and a predicate represents a binary relation between elements of two classes. Ontologies have been successfully applied in a number of domains to facilitate the representation, inference and integration of knowledge and data (Ruttenberg et al., 2009; Smith et al., 2007; Bizer et al., 2009).

As ontology use has become more prevalent, the number and size of ontologies has increased rapidly. Some of the largest ontologies-such as SNOMED CT (Stearns et al., 2001), the Galen Ontology (Rogers and Rector, 1996) and the Gene Ontology (Ashburner et al., 2000) — contain tens and even hundreds of thousands of classes and predicates. This makes understanding, maintenance and application of them a major challenge. New methods and systems are needed that support effective interrogation and comprehension of such large ontologies.

When understanding a large ontology two of the most fundamental tasks are: (1) find a particular class of interest and understand its relationship with other classes in the ontology, and (2) understand the overall structure of the ontology, in particular the taxonomy of classes and properties, i.e., their inheritance hierarchy.

Information visualization techniques have been applied to help with these tasks, with a large number of different approaches proposed over the years (Katifori et al., 2007; Lanzenberger et al., 2010). These approaches include 2D and 3D visualization, and range from simple indented list and tree-based layout methods to space filling and distortion based techniques. However, it is fair to say that none of the existing techniques or systems are adequate.

In their comprehensive survey (Katifori et al., 2007), Katifori et. al. point out a number of common deficiencies of current systems. These include:

Clutter - that the visualization produced is messy and not easy to make sense of, and made worse with the increase in ontology size,

Structure - the visualization does not effectively show the overall hierarchical structure of the ontology taxonomy,

Scalability - medium and large ontologies are not handled efficiently, and

Inspection - it is difficult to interactively inspect and interrogate the ontology in different ways, including search, highlight and querying.

In this paper, we tackle the problem of visualizing large OWL (Horrocks et al., 2003) and OWL 2 (Grau et al., 2008) ontologies and present a novel visualization method and system that address the deficiencies identified above. Our main contributions are twofold: 
- The proposal of a novel tri-view landmark+local+axiom ontology visualization model that addresses the problems of clutter and structure.

- The development of a scalable and feature-rich ontology visualization engine that supports this model. The engine implements efficient, automatic graph layout algorithms and is capable of visualizing and interrogating large ontologies efficiently, hence addressing the issues of scalability and inspection.

Our visualization model consists of three separate views that allow the user to visualize classes and properties in an OWL ontology in increasing granularity: landmark view, local view and axiom view. Firstly, an orthogonal tree-based landmark view of the class (resp. property) hierarchy is maintained. This landmark view provides a quick global perspective for the entire ontology. Since it is infeasible to show all classes for large ontologies, this view displays a simplified hierarchy using a number of inferred and userdefined landmark or key classes.

Secondly, a local view presents the full inheritance hierarchy for a small number of classes (resp. properties). The user can browse within this view by expanding and collapsing branches of hierarchy, and by changing the particular class (resp. properties) on which the local view is focused.

Thirdly, an axiom view shows the OWL axioms of a particular selected class (resp. property) and the associated anonymous expressions using a nested rectangles visualization based on the expression syntax.

We have implemented our ontology visualization method as a plugin for the Dunnart (Dwyer et al., 2008) constraint-based diagram editor. A screenshot of the system visualizing the well-known Galen Ontology (Rogers and Rector, 1996) can be seen in Figure 1 . Note that Galen is a large ontology that contains more than 23,000 classes and 63,000 axioms.

The visualization engine includes a number of novel features:

- Efficient, automatic layered tree-like layout of the inheritance hierarchies in the landmark view and local view.

- Synchronized, bidirectional navigation between the landmark view and local view.

- Automatic selection and dynamic update of the landmark classes (resp. properties) in the landmark view.

- Flexible search and highlighting capabilities.

- Fast loading of large ontologies via a database backend.
- Natural visualization of anonymous class (property) expressions and axioms in the axiom view.

The rest of the paper is organized as follows. Section 2 briefly surveys relevant works. Section 3 discusses our landmark+local+axiom visualization approach, including a detailed description of the system functionality and performance. Section 4 concludes the paper and presents directions for future work.

\section{Background}

\subsection{Semantic Web Ontologies}

Mainstream ontology languages such as OWL (Web Ontology Language) (Horrocks et al., 2003) and OWL 2 (Grau et al., 2008) are defined based on expressive description logics (Baader and Nutt, 2003).

The most important kind of object in an ontology are the classes. These are organized in an inheritance hierarchy called the class taxonomy. A key requirement of ontology visualization is to be able to understand this taxonomy. Ontologies may contain multiple inheritance so this is not a true hierarchy but rather a DAG (directed acyclic graph). While inheritance is the primary relationship relating classes, an ontology language also includes a number of language constructs that can be used to construct complex expressions and axioms to describe and relate classes, predicates and individuals using other properties.

For example, the Campylobacter class in the Galen Ontoolgy (shown in both Figure 1 and Figure 2) is defined with the following axioms and class expressions (in the OWL Manchester syntax):

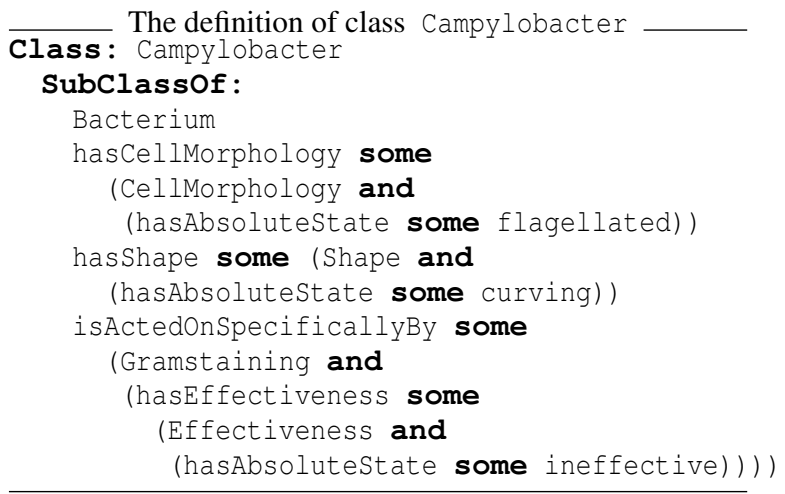

In the above ontology snippet an OWL class, Campylobacter, is being defined. The SubClassof axioms define four super classes for this class, three of which are anonymous, nested class expressions that restrict the membership of this class. Such class expressions include existential quantification and class conjunction.

The semantics of an ontology is based on its interpretation I (Baader and Nutt, 2003). A class C 


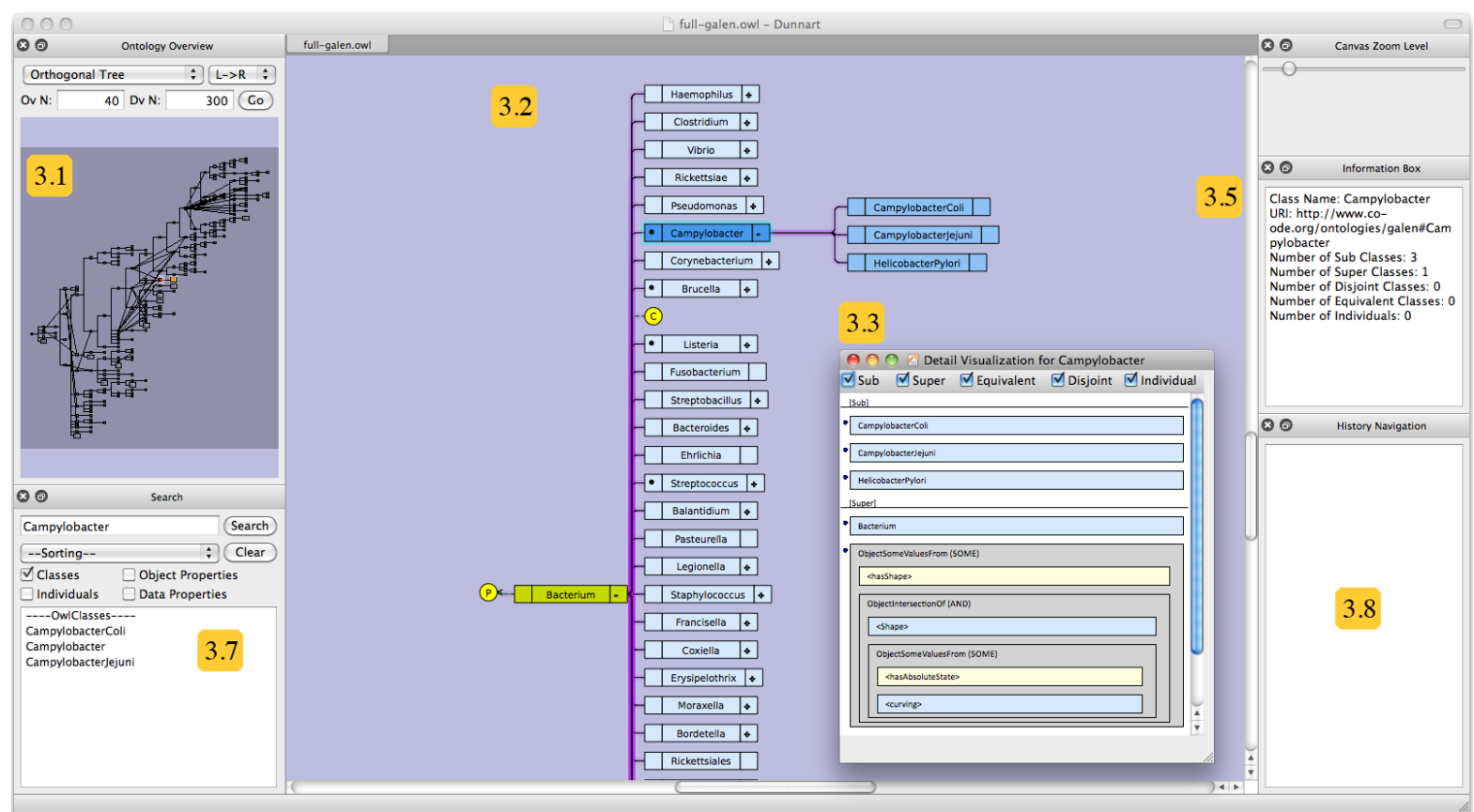

Figure 1: Screenshot of our system being used to visualize the Galen Ontology. The landmark view is shown on the left (Section 3.1), the local view in the center (Section 3.2), and the axiom view for one class (Campylobacter) in the inset window (Section 3.3). The information pane of the class Campylobacter is shown in top right, below the canvas zoom control (Section 3.5). Search and highlighting can be performed in the bottom left panel (Section 3.7), and history navigation is show in bottom right (Section 3.8).

is interpreted (denoted $\mathrm{C}^{I}$ ) as a set of individuals. A predicate $\mathrm{p}$ is interpreted (denoted $\mathrm{p}^{I}$ ) as a set of pairs $\langle x, y\rangle$ that are mapped under $\mathrm{p}$. The interpretationbased semantics of the axioms and expressions used in the above example can be found in Table 1 below. Table 1: Semantics of some OWL axioms and definitions.

\begin{tabular}{l|l}
\hline Axiom/Expression & definition \\
\hline $\mathrm{A}$ SubClassof : $\mathrm{B}$ & $\mathrm{A}^{I} \subseteq \mathrm{B}^{I}$ \\
$\mathrm{~A}$ and B & $\mathrm{A}^{I} \cap \mathrm{B}^{I}$ \\
$\mathrm{p}$ some C & $\left\{x \mid \exists y .\langle x, y\rangle \in \mathrm{p}^{I} \wedge y \in \mathrm{C}^{I}\right\}$ \\
\hline
\end{tabular}

The number and size of ontologies have been growing steadily over the past decade with the increased adoption of the Semantic Web and Linked Data (Bizer et al., 2009) techniques. In domains like bioinformatics, a number of large ontologies have been used as an important tool for the task of capturing domain knowledge and facilitating knowledge exchange and data integration (Ruttenberg et al., 2009; Smith et al., 2007).

Some well-known and widely-used ones that are available on the BioPortal ontology repository ${ }^{1}$ include the Galen ontology, the Gene Ontology, the NCBI organismal classification ontology, the NCI

\footnotetext{
$1_{\text {http: / / bioportal.bioontology.org/ }}$ (accessed 2012-11-28)
}

Thesaurus ontology and the SNOMED CT (clinical terms) ontology. These large ontologies contain tens of thousands up to almost a million classes. Thus, there is a pressing need for visualization systems to assist in the understanding and maintenance of such large ontologies.

\subsection{Ontology Visualization}

Quite a number of visualization techniques have been employed in different systems to facilitate the navigation and interrogation of OWL and RDFS ontologies. A number of ontology visualization systems are catalogued in (Lanzenberger et al., 2010), with a focus on tools that support ontology mapping and alignment. A comprehensive survey (Katifori et al., 2007) categories these systems according to their visualization approaches. The survey also critically analyses the visualization systems, and identifies a number of common flaws. As discussed in Section 1, these shortcomings can be grouped into the following categories: clutter, structure, scalability and inspection.

In the rest of this subsection, we briefly introduce the main visualization approaches and discuss their advantages and shortcomings.

Indented list is a basic, textual visualization approach that arranges classes (resp. properties) in a tree view. This approach is employed by most ontology editors, including the popular Protégé ontology edi- 


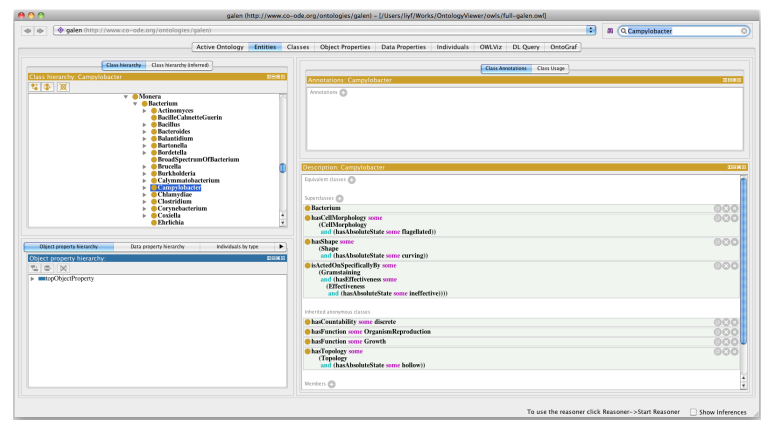

(a) Protégé's visualization of the Galen Ontology, showing the axioms of the Campylobacter class.

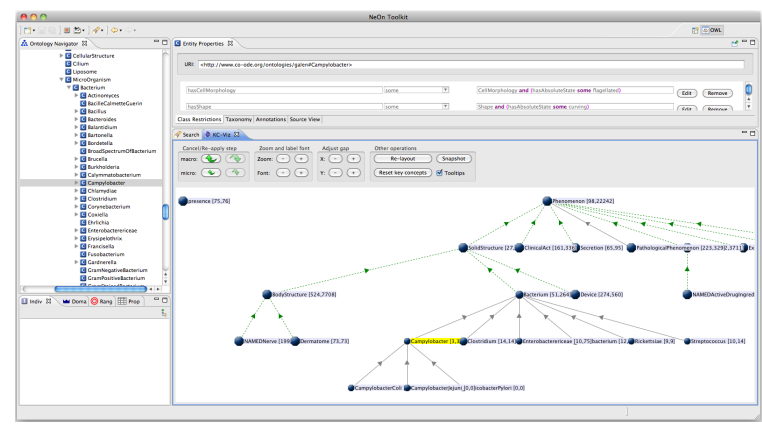

(b) KC-Viz's visualization of the Galen Ontology, showing the axioms of the Campylobacter class.

Figure 2: Visualization of the Galen Ontology produced by Protégé and KC-Viz, focused on the same OWL class, Campylobacter, as in Figure 1.

tor (shown in Figure 2(a)). The main advantages of this approach includes intuitiveness, familiarity, nonoverlapping of labels and fast browsing.

A disadvantage is that indented lists are not well suited to handling multiple inheritance. However, since multiple inheritance is not that common, some ontology visualization tools, such as Protégé, ${ }^{2}$ using representations designed for hierarchical data handle multiple inheritance by duplicating the concept and its sub-concepts in the hierarchy under each of its parents. We believe such duplication is confusing to the user and leads to an unnecessary increase in the size of the visualization.

Lack of an overview and the poor use of space have also been identified as shortcomings of indented lists. Nevertheless, the indented list is recognized as a baseline visualization approach that can be combined with other techniques. Note that in Figure 2(a) only a small portion of the hierarchy is shown in the indented list on the left. Hence, indented lists suffer from the issues of structure.

Node-link graphs and trees are another intuitive approach to visualizing hierarchies where nodes represent classes and edges represent inheritance relationships. It naturally captures the usually hierarchical inheritance relationship among classes in an ontology and for moderately sized networks it can, at least in principle, clearly show the sub-class relationship and the class structure. The main disadvantage is that it is not a particularly compact representation and networks with more than a few hundred textually labelled nodes are not really understandable when viewed on a standard screen. Also, depending upon the layout method, there may be efficiency issues for large ontologies. Hence applications, including On-

\footnotetext{
${ }^{2}$ http: //protege.stanford.edu/ (accessed 2012-11-28)
}

toViz, ${ }^{3}$ that employ such an approach suffer from clutter and inspection issues (Katifori et al., 2006).

Zoomable (nested) approaches such as Jambalaya ${ }^{4}$ and CropCircles ${ }^{5}$ place child nodes inside their parents and allow nodes to be zoomed in and out. Being effective in locating specific nodes, zoomable approaches have difficulty in handling labels and maintaining a global overview. Such nested visualization systems therefore face clutter and structure issues. Also, in the case of multiple inheritance they require duplication of a concept and its sub-concepts in the hierarchy under each of its parents.

Space-filling techniques aim at making full use of the display area by filling the space with nodes. A node's display area is subdivided among its descendant nodes, with the size of a node allocated in proportion to the node's size, number of child nodes, etc. Systems based on this technique, such as Treemap, ${ }^{6}$ are effective in visualizing ontologies with a shallow inheritance hierarchy (2 to 3 levels). However, they are not as effective in presenting the structure of the ontology. Similarly, they suffer from the issue of structure.

Focus + context or distortion techniques allow the view to be distorted to focus on a particular node, while shrinking the other nodes. Such approaches, including Hyperbolic Tree Visualization, ${ }^{7}$ facilitate easy navigation in the hierarchy while maintaining

\footnotetext{
3 http: //protegewiki.stanford.edu/wiki/ OntoViz (accessed 2012-11-28)

4 http: //thechiselgroup.org/2004/07/06/ jambalaya/ (accessed 2012-11-28)

5 http: //www.mindswap.org/2005/cropcircles/ (accessed 2012-11-28)

$6_{\text {http: }} / /$ www.cs.umd.edu/hcil/treemap/ (accessed 2012-11-28)

${ }^{7}$ http: // jowl.ontologyonline.org/ HyperBolicTree.html (accessed 2012-11-28)
} 
contextual information (neighbor nodes). However, hierarchies, an important concept in ontologies, are not easily shown in such approaches. These systems face the problem of structure.

Except Protégé, all of the above approaches focus on showing the structure of the ontologies, but not on showing the details of individual classes (properties). Hence, they all face the problem of inspection. Protégé, being an ontology editor, combines the indented list view with a panel that shows the details of individual nodes in the list. The details of a class are shown in the bottom right panel in Figure 2(a).

More recently, concept diagrams have been applied to visualize a number of different relations of a group of classes and properties (Howse et al., 2011). Visual notations are defined for various ontological axioms and expressions such as class disjointness, equivalence and all values from. However, this proposed visualization method has not been implemented yet and it is unclear how effective it will be in handling large groups of classes and properties.

Other recent works including (da Silva and Freitas, 2011; Vercruysse et al., 2012) face similar issues to those outlined above.

Recently, KC-Viz (Motta et al., 2011), a nodelink-based ontology visualization tool has been developed as part of the $\mathrm{NeON}$ project. ${ }^{8} \mathrm{KC}$-Viz uses a hybrid representation of an indented list for class listing and a node-link graph for local structure. The node-link graph is initialized with a number of key concepts, concepts that are deemed to be important by some metrics, to provide an initial, quick summary of the entire ontology. These behave like landmarks. However, an overview containing just the landmarks is not preserved during interaction. During exploration the node-link graph is expanded as non-key concepts are explored. The key concepts are not visually distinguished from regular, non-key concepts. Thus the utility of the key concept view is limited once more concepts are added to the visualization. The structure and clutter issues intrinsic to the indented list and node-link graph methods are also present in KC-Viz. KC-Viz's visualization of the Campylobacter class in the Galen Ontology can be found in Figure 2(b).

Observing that only a handful of visualization systems are capable of handling ontologies with more than 100,000 nodes, it is concluded (Katifori et al., 2007) that scalability is an important issue in ontology visualization.

From the above discussion it can be clearly seen that no single visualization approach effectively ad-

\footnotetext{
8 http: / / www . neon-foundation.org/ (accessed 2012-11-28)
}

dresses the four deficiencies we identified above. This observation motivated us to propose a novel tri-view visualization approach that alleviates such problems.

\section{Ontology Visualization with Land- mark+Local+Axiom Views}

From the last section, it is clear that a key problem in ontology visualization is how to provide a meaningful overview of the class taxonomy for large ontologies that can be computed efficiently and which is smoothly integrated with detailed information about a particular class. While many previous approaches have used techniques designed for single inheritance hierarchies, we believe that it is important to use a visualization that can naturally handle DAGs arising because of multiple inheritance. The most common representation for a DAG in information visualization applications is a hierarchical or layered tree-like network diagram. However, as we have discussed, while well suited to showing taxonomies with a few hundred nodes it does not scale to larger taxonomies.

Because of the very large number of classes in large taxonomies we do not believe it makes sense to show all of the classes at once. These taxonomies are so large that even if each class uses only a few pixels, this will take up most of the screen space. We believe a better approach is to use representative classes in the taxonomy to provide an overview. These representative classes act as landmarks for navigation, and the position of individual classes in the taxonomy is understood w.r.t. the landmark classes in the overview. The use of a landmark overview for navigating in large networks has previously been used for visualizing the structure of large websites (Mukherjea and Hara, 1997). In a closely related idea, the papers (Peroni et al., 2008; Motta et al., 2011) suggest providing an abstraction of the taxonomy by showing the sub-taxonomy for selected key concepts. However, as discussed in the previous section, the design of their visualization model differs significantly from ours.

In this section we detail our novel tri-view approach to ontology visualization including the interaction model and the interesting implementation issues we encountered when building a system based on the model. The model has three kinds of views: the landmark view which provides an overview of the structure of the class (or property) taxonomy by showing a sub-taxonomy for landmarks which comprise automatically identified key concepts as well as user-specified landmarks; a local view that shows all concepts in some sub-network of the taxonomy. Both the landmark and local view of the taxonomy use a layered network representation. The final view, the 
axiom view details the properties associated with a class. The user can open multiple local and axiom views with different focal areas, if they desire.

\subsection{Landmark View}

The landmark view consists of the sub-hierarchy connecting the landmark nodes. The first time that an ontology is viewed with our tool the initial landmarks must be determined. This is relatively costly. However as the landmarks are stored between sessions this needs only be done once for that ontology.

Our starting point for finding the $n$ key concepts for the landmark view is the algorithm given in Peroni et al. (Peroni et al., 2008). This uses a set of static measures to calculate a score for each concept in ontologies. These measures include natural categories, density, coverage, and popularity. Natural categories aim to identify concepts that are informationrich in a psycho-linguistic sense. Density highlights the information-rich concepts in an ontological sense. The popularity criterion aims to identify concepts that are particularly common. Coverage is used to ensure that no important parts are ignored. We found that this algorithm was very slow for large ontologies and modified it as follows: we no longer check for coverage as this was expensive and in practice makes little difference; rather than performing a Google search to find out how popular a particular concept is, we use the number of times it appears in the ontology axioms.

However this static measure is only the initial determination: the choice of key concepts evolves dynamically as a result of user interaction. The number of times the concept is selected in either the landmark view or local view is tallied to give a good indication of its importance. Furthermore, we allow the user to directly promote or demote concepts to the landmark view. The final importance score for each concept is computed by combining the static and dynamic scores, then sort the scores and obtains the list of key concepts.

A subontology for the key concepts is then extracted. Multiple inheritance in the ontology is removed from the subontology by first computing the depth of each concept from the root and then treating a concept with multiple parents as the child of its deepest parent. We compute the layout using Walker's layered tree layout algorithm with the modifications to ensure linearity (Walker, 1990; Buchheim et al., 2002). Finally, the removed parent-child edges are reintroduced into the tree once we have computed the layout, as shown in Figure 3. Organizing nodes based on their lexicographical ordering has been shown to improve the usability of graphs. This is the reason we use a modification of Walker's algorithm rather than a standard DAG layout algorithm such as Sugiyama

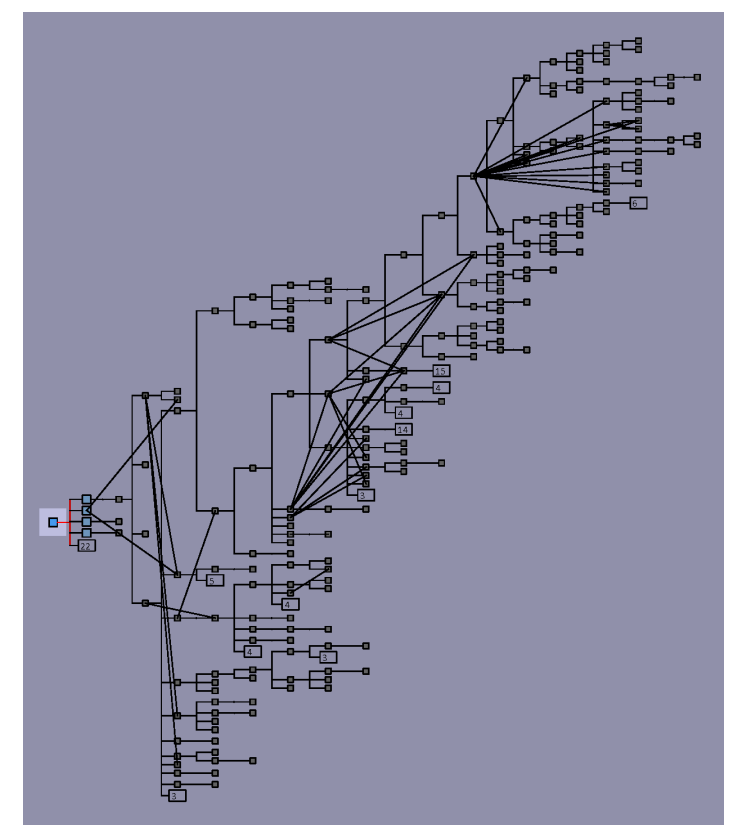

Figure 3: Screenshot of landmark view (Galen Ontology). Non-orthogonal edges indicate multiple inheritance.

layered layout (Sugiyama et al., 1981) which reorders nodes within levels.

We also perform one additional simplification of the resulting tree. When there are multiple leaf nodes (nodes without any children) of a single parent above some threshold, we collapse these down to a single larger node in the landmark view. The main reason for this is to make better use of space and to prevent particularly broad trees from being very long in the $x$ or $y$ dimension.

The landmark tree is scaled uniformly to fit within the landmark pane of the interface, which is can itself be resized and repositioned by the user. When the user hovers over nodes in this view, a tooltip will show the name of the concept beneath the cursor. Clicking anywhere in this view chooses a new focal area based on the landmarks in closest proximity to the mouse cursor. A square highlight is drawn around landmarks that are part of the current focal area, as shown with the root node of the tree in Figure 3.

\subsection{Local View}

The local view shows a subset of the taxonomy, which we refer to as the focal area. This is a userconfigurable amount of concepts, but we prefer a default of 40 nodes to keep the network understandable and manageable. The local view is centered around a single focal concept, either selected by the user clicking in the local view, a search result, or the closest concept to the point they clicked in the landmark 
view. In order to understand the relationship between the local view and the landmark view, this node, its parents and children are shown with a distinct color coding in both views, as shown in the center pane of Figure 1. In both views, the focal node Thing are coded in darker blue whereas its child nodes are coded in lighter blue. If the focal concept is not a landmark then the closest landmark to it (graph theoretically) is color coded in the landmark view. In addition, if the user brushes (hovers their mouse) over any concept in the local view, a red circle is momentarily drawn around the closest corresponding concept in the landmark view.

We use constrained network layout (Dwyer et al., 2006) together with orthogonal edge routing (Wybrow et al., 2010) to perform the layout of the local view. For the purpose of layout, we consider the network in the local view to be a tree, discarding edges causing multiple inheritance. We then traverse this tree and assign a level for each node based on its distance from the root node. Next we perform forcedirected layout, constraining nodes on each level to be aligned with each other, and ordering between levels (causing the tree to flow in a particular direction). The initial force-directed aspect of the layout causes leaves from separate branches to move apart from each other and not be intertwined (see center pane of Figure 1). Constraints are effectively used to ensure that the layout is layered and that the relative placement of concepts shared between the landmark view and local view is the same. We also apply additional constraints to prevent nodes from overlapping. This ensures that their labels remain readable.

Edges between nodes are drawn as orthogonal routes made of horizontal and vertical line segments with rounded corners. We position the middle segment of edges in the channel bordered by the largest nodes in each level. We also adjust the position within this channel to distinguish between bundles of edges connecting common parents or children, as can be seen between the middle levels in Figure 1.

\subsection{Axiom View}

The concept taxonomy, while important, is not the only relationship between concepts. A separate $a x$ iom view window shows the axioms associated with the focal concept. More exactly this shows the sub, super-, equivalent-, and disjoint-class axioms that directly refer to that concept. Figure 4 shows this window for the Campylobacter class, with some sub classes and (anonymous) super classes. The user can choose to show the formula textually or using nested rectangles based on the syntax similar to the representation used in Xing for XML queries in (Erwig, 2003).

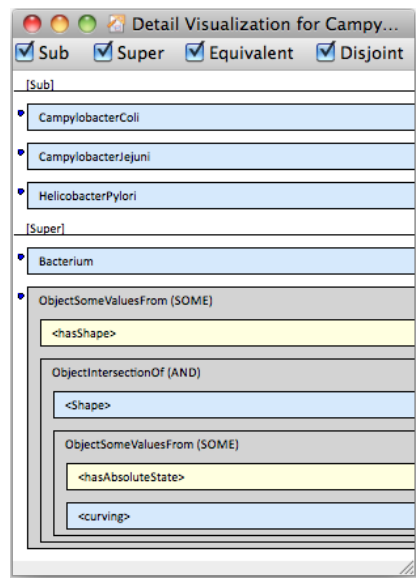

Figure 4: Screenshot of the Axiom view for the Campylobacter class (Galen Ontology).

\subsection{Viewing Properties}

The properties in the ontology are presented in a similar way to the concepts: a separate property local view shows the taxonomy while a property axiom view shows the domain and range of a selected property and the properties it is associated with. In theory another landmark view could be provided for properties but in practice we have found that the complete property taxonomy can usually be shown in the local view.

\subsection{Information Pane}

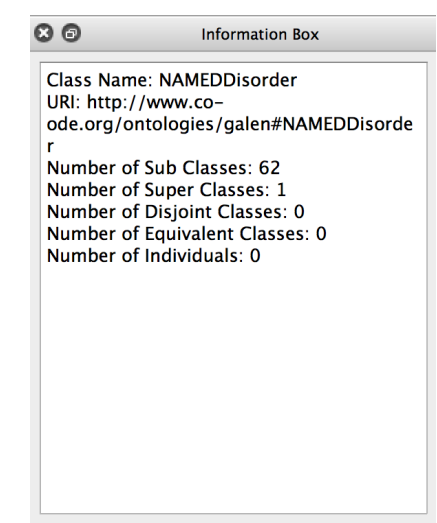

Figure 5: Screenshot of Information Pane (Galen Ontology).

An information pane shows textual information about the current focal concept, as shown in Figure 5. We use it to show statistics about the focal concept, and information that is difficult to display elsewhere in the visualization, such as the fully qualified URI of the concept.

\subsection{Navigation}

The effectiveness of any ontology visualization tool depends crucially on how easy it is to navigate 


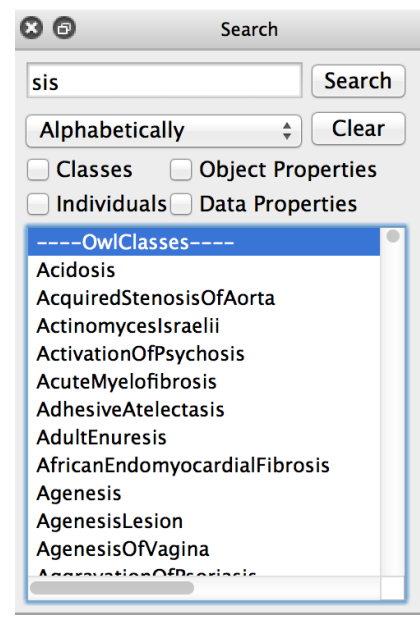

Figure 6: Screenshot of Search Pane (Galen Ontology).

through the ontology to find the detailed information the user is interested in while still keeping track of where they are in the ontology.

The local view is the main way of viewing concepts in the ontology. A new area of focus is triggered by the user double-clicking on a particular concept in the landmark view, local view, axiom view, property view or search results list. This selected concept is placed in the middle of the local view and its immediate ancestors and descendants are shown with it along with the closest key concepts from the landmark view. Each node is displayed with a box at either end. Where the concept has ancestors or descendants, the node will show a "+" or "-" symbol that the user can click to show or hide its parents or children. The changes to the layout due to nodes being added or removed is smoothly animated. This allows the user to explore the neighborhood surrounding the concepts initially chosen to be displayed in the local view.

The user can promote a concept in the local view to the landmark view. When this happens the layout for the landmark view is recomputed and the layout changes are smoothly animated. This is useful if the user wishes to provide their own points of interest, or wish to augment the inferred key concepts with their own prior knowledge.

\subsection{Search and Highlighting}

The tool provides a powerful search mechanism. This is accessible to the user via the Search pane, as shown in Figure 6. The user can enter a search term. The system will return a list of classes, individuals and properties that match the user's search term. All these matches will be highlighted in both the landmark view (see for example Figure 7) and the local view. The user can hover their mouse cursor over any item in the

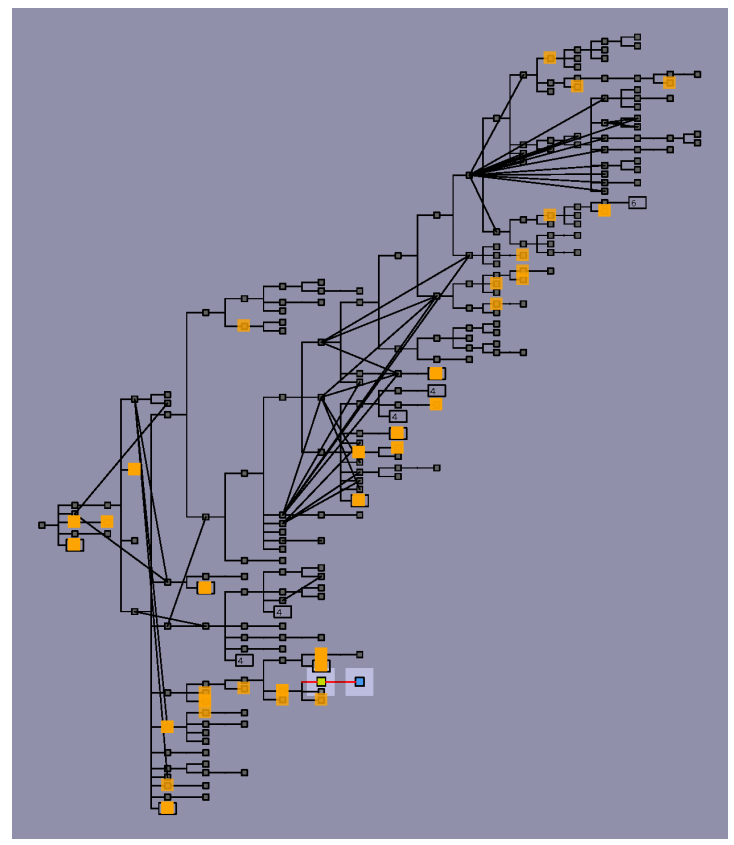

Figure 7: Screenshot of Landmark View with highlighted search results (Galen Ontology).

search results list to cause only that item to be highlighted in the landmark and local view (or the closest concept to it in the case of the landmark view). The user may click on any item in the search results list to make that the focal concept and cause the local view to update to a new focal area. This dual search design allows the user to find and select any concept within the entire ontology but can also be used to effectively search and highlight information already shown in the local view without causing that view to switch to a different focal area.

\subsection{Navigation History}

The system also offers a Navigation History pane. This pane shows an ordered list of concepts selected via the landmark view, local view, axiom view, property view or search results list when determining a new focal area for the local view. This allows the users to quickly navigate back to previous areas of interest within the local view.

\subsection{Performance}

A typical problem with existing ontology visualization tools and editors is slow loading times. This is predominantly due to the OWL standard for ontology specification being an XML-based language and the default technique for reading it being via the Java OWLAPI. We found this to be prohibitively slow for loading large ontologies (ten to fifteen minutes to read the entire Galen Ontology and more than thirty minutes to read the Gene Ontology). Thus, we believe 
Table 2: Loading times for various ontologies (in msec.) from a database.

\begin{tabular}{|l|r|l|r|r|r|r|}
\hline Name & Nodes & First load & Ontology data & Key concepts & Drawing & Total \\
\hline ICNP & 3,291 & yes & 1,489 & 27,700 & 641 & 29,830 \\
& & no & 1,479 & 266 & 748 & 2,493 \\
\hline Galen Ontology & 23,142 & yes & 7,214 & 636,541 & 822 & 644,577 \\
& & no & 7,075 & 7,459 & 696 & 15,230 \\
\hline Gene Ontology & 35,228 & yes & 11,215 & $1,603,332$ & 2,180 & $1,616,727$ \\
& & no & 10,751 & 96,919 & 2,797 & 110,467 \\
\hline
\end{tabular}

that it makes much more sense to use a database to store the ontology and efficiently load sections of it as required. The database can then be optimized for the particular queries that individual systems require.

We have developed a translation tool that reads an OWL ontology and stores its contents in a SQLite ${ }^{9}$ database. Our ontology visualization engine can subsequently read the ontology data from the database much more quickly. The calculation of key concepts is also computationally expensive. Hence, the key concepts are stored in the database to speed up subsequent loading by avoiding recalculation. Table 2 shows some time measurement (in msec.) for loading and working with various-sized real-world ontologies from database. It can be observed that for mediumto large-sized ontologies, our database-based loading approach (second row for each ontology) achieves a dramatic performance improvement of up to 40-fold.

\section{Conclusion}

Semantic Web ontologies are expressive taxonomies in which concepts may also have complex relationships with each other. Many ontologies have been developed for the tasks of knowledge representation and data annotation in various application domains. Hence, the understanding of such ontologies is crucial to their maintenance and deployment.

Ontology visualization has been recognized as an important tool to improve their navigation and interrogation, especially for large and complex ontologies. Many ontology visualization system has been proposed and developed. However, as surveyed in (Katifori et al., 2007) and elsewhere, existing systems suffer from various deficiencies in the categories of: clutter, structure, scalability and inspection.

In this paper, we present a novel tri-view ontology visualization method addresses the above deficiencies. The proposed method is backed by an implementation that supports interactive visualization and is capable of handling large ontologies effectively. Our main contributions can be summarized as follows:

\footnotetext{
${ }^{9}$ http://www.sqlite.org/ (accessed 2012-11-28)
}

- The proposal of a novel tri-view ontology visualization method that presents the ontology in increasing granularity.

The landmark view is separate and persistent and presents a global perspective of the ontology's inheritance hierarchy. The local view shows in more detail the connections between a small group of concepts. The axiom view shows the axioms of a selected concept.

- An implementation that can efficiently handle large ontologies such as the Galen Ontology (23,000 concepts) and Gene Ontology $(35,000$ concepts). Our implementation also includes a number of practical features such as search and highlighting that improve the usability of the system.

We believe that our visualization method and implementation alleviate the four deficiencies we identify above, and hence represents a novel contribution to the problem of large ontology visualization.

A number of future work directions have been identified. We will further improve the efficiency of the system. Specifically, we will improve the calculation of landmarks by incorporating metrics that are easy to calculate (such as the ones introduced in (Zhang et al., 2010; Kang et al., 2012)) and optimize the calculation algorithm. The layout algorithms will also be further analyzed and improved. Moreover, the definition of key concepts will be examined to develop a way to better ensure preservation of the ontology's overall structure in the landmark view.

Besides, formal usability tests will be conducted to rigorously evaluate the effectiveness of the system. Advanced features such as integration with ontology reasoners and supporting editing capabilities are also planned.

\section{Acknowledgments.}

NICTA is funded by the Australian Government as represented by the Department of Broadband, Communications and the Digital Economy and the Australian Research Council. We acknowledge the support of the ARC through Discovery Project Grant DP0987168 and DP110101390. 


\section{REFERENCES}

Aroyo, L., Welty, C., Alani, H., Taylor, J., Bernstein, A., Kagal, L., Noy, N. F., and Blomqvist, E., editors (2011). The Semantic Web - ISWC 2011 - 10th International Semantic Web Conference, Bonn, Germany, October 23-27, 2011, Proceedings, Part I, volume 7031 of Lecture Notes in Computer Science. Springer.

Ashburner, M., Ball, C. A., Blake, J. A., et al. (2000). Gene Ontology: Tool for the Unification of Biology. Nat Genet, 25(1):25-29.

Baader, F. and Nutt, W. (2003). Basic description logics. In Baader, F., Calvanese, D., McGuinness, D., Nardi, D., and Patel-Schneider, P., editors, The description logic handbook: theory, implementation, and applications, pages 43-95. Cambridge University Press.

Bizer, C., Heath, T., and Berners-Lee, T. (2009). Linked data - The story so far. International Journal on Semantic Web and Information Systems (IJSWIS), 5(3): $1-22$.

Buchheim, C., Jünger, M., and Leipert, S. (2002). Improving Walker's algorithm to run in linear time. In Graph Drawing, pages 347-364. Springer.

da Silva, I. C. S. and Freitas, C. M. D. S. (2011). Using visualization for exploring relationships between concepts in ontologies. In Proceedings of the 2011 15th International Conference on Information Visualisation (IV'11), pages 317-322. IEEE Computer Society.

Dwyer, T., Koren, Y., and Marriott, K. (2006). Ipsepcola: An incremental procedure for separation constraint layout of graphs. Visualization and Computer Graphics, IEEE Transactions on, 12(5):821-828.

Dwyer, T., Marriott, K., and Wybrow, M. (2008). Dunnart: A constraint-based network diagram authoring tool. In Tollis, I. G. and Patrignani, M., editors, Graph Drawing, volume 5417 of Lecture Notes in Computer Science, pages 420-431. Springer.

Erwig, M. (2003). Xing: A visual xml query language. Journal of Visual Languages \& Computing, 14(1):545.

Grau, B. C., Horrocks, I., Motik, B., Parsia, B., PatelSchneider, P., and Sattler, U. (2008). OWL 2: The next step for OWL. Journal of Web Semantics: Science, Services and Agents on the World Wide Web, 6:309-322.

Horrocks, I., Patel-Schneider, P. F., and van Harmelen, F. (2003). From $\mathcal{S H} I Q$ and RDF to OWL: The Making of a Web Ontology Language. Journal of Web Semantics, 1(1):7-26.

Howse, J., Stapleton, G., Taylor, K., and Chapman, P. (2011). Visualizing ontologies: A case study. In (Aroyo et al., 2011), pages 257-272.

Kang, Y.-B., Li, Y.-F., and Krishnaswamy, S. (2012). Predicting reasoning performance using ontology metrics. In Cudré-Mauroux, P., Heflin, J., Sirin, E., Tudorache, T., Euzenat, J., Hauswirth, M., Parreira, J. X., Hendler, J., Schreiber, G., Bernstein, A., and Blomqvist, E., editors, International Semantic Web Conference (1), volume 7649 of Lecture Notes in Computer Science, pages 198-214. Springer.
Katifori, A., Halatsis, C., Lepouras, G., Vassilakis, C., and Giannopoulou, E. G. (2007). Ontology visualization methods - a survey. ACM Comput. Surv., 39(4).

Katifori, A., Torou, E., Halatsis, C., Lepouras, G., and Vassilakis, C. (2006). A Comparative Study of Four Ontology Visualization Techniques in Protégé: Experiment Setup and Preliminary Results. In Information Visualization, 2006. IV 2006. Tenth International Conference on, pages $417-423$.

Lanzenberger, M., Sampson, J., and Rester, M. (2010). Ontology visualization: Tools and techniques for visual representation of semi-structured meta-data. J. UCS, 16(7):1036-1054.

Motta, E., Mulholland, P., Peroni, S., d'Aquin, M., GómezPérez, J. M., Mendez, V., and Zablith, F. (2011). A novel approach to visualizing and navigating ontologies. In (Aroyo et al., 2011), pages 470-486.

Mukherjea, S. and Hara, Y. (1997). Focus+ context views of world-wide web nodes. In Proceedings of the eighth ACM conference on Hypertext, pages 187-196. ACM.

Peroni, S., Motta, E., and d'Aquin, M. (2008). Identifying key concepts in an ontology, through the integration of cognitive principles with statistical and topological measures. In Domingue, J. and Anutariya, C., editors, ASWC, volume 5367 of Lecture Notes in Computer Science, pages 242-256. Springer.

Rogers, J. and Rector, A. (1996). The galen ontology. In Proceedings of Medical Informatics Europe (MIE 96), pages 174-178. IOS Press.

Ruttenberg, A., Rees, J., Samwald, M., and Marshall, M. S. (2009). Life Sciences on the Semantic Web: the Neurocommons and Beyond. Briefings in Bioinformatics, 10(2):193-204.

Smith, B. et al. (2007). The OBO Foundry: Coordinated Evolution of Ontologies to Support Biomedical Data Integration. Nat Biotech, 25(11):1251-1255.

Stearns, M. Q., Price, C., Spackman, K. A., and Wang, A. Y. (2001). SNOMED clinical terms: overview of the development process and project status. In Proc AMIA Symp, pages 662-666, College of American Pathologists, Northfield, IL, USA.

Sugiyama, K., Tagawa, S., and Toda, M. (1981). Methods for visual understanding of hierarchical system structures. IEEE Transactions on Systems, Man, and Cybernetics, 11(2):109-125.

Vercruysse, S., Venkatesan, A., and Kuiper, M. (2012). OLSVis: an animated, interactive visual browser for bio-ontologies. BMC Bioinformatics, 13(1):116+.

Walker, J. Q. (1990). A node-positioning algorithm for general trees. Software Practice and Experience, 20(7):685-705.

Wybrow, M., Marriott, K., and Stuckey, P. (2010). Orthogonal connector routing. In Graph Drawing, pages 219231. Springer.

Zhang, H., Li, Y.-F., and Tan, H. B. K. (2010). Measuring Design Complexity of Semantic Web Ontologies. Journal of Systems and Software, 83(5):803-814. 